Article

\title{
Linear Sensitivity Modelling Useful for Voltage Control Analysis Using Power Injections from DER
}

\author{
Ulises D. Lubo-Matallana *(D), Miguel Ángel Zorrozua and José Félix Miñambres \\ Department of Electrical Engineering, Faculty of Engineering Bilbao, University of the Basque \\ Country (UPV /EHU), Plaza Ingeniero Torres Quevedo 1, 48013 Bilbao, Spain; \\ miguelangel.zorrozua@ehu.eus (M.Á.Z.); josefelix.minambres@ehu.eus (J.F.M.) \\ * Correspondence: ulubo001@ikasle.ehu.eus; Tel.: +34-946017278
}

check for updates

Citation: Lubo-Matallana, U.D.; Zorrozua, M.Á.; Miñambres, J.F. Linear Sensitivity Modelling Useful for Voltage Control Analysis Using Power Injections from DER. Energies 2021, 14, 4749. https://doi.org/ $10.3390 /$ en14164749

Academic Editor: Seon-Ju Ahn

Received: 28 May 2021

Accepted: 26 July 2021

Published: 4 August 2021

Publisher's Note: MDPI stays neutral with regard to jurisdictional claims in published maps and institutional affiliations.

Copyright: (c) 2021 by the authors. Licensee MDPI, Basel, Switzerland. This article is an open access article distributed under the terms and conditions of the Creative Commons Attribution (CC BY) license (https:// creativecommons.org/licenses/by/ $4.0 /)$.

\begin{abstract}
The injection of apparent power to self-consumption buses generates voltage variations during network operation, which, when properly monitored, could support voltage regulation and control. In this paper, we propose a linear sensitivity modelling, quite useful for studies of voltage regulation with distributed energy resources (DER). This modelling consists of two analytical improvement steps: first, a full formulation for the voltage deviations, and second, the influence of line capacitance as $Q$-injections at the points of common couplings (PCCs). Our proposal is based on the linear topological sensitivity of an existing network (as a function of the line parameters $X, R$, and $B c$ ), branch power flow (active-reactive power $(P-Q)$ ), and power injections at the PCCs. Here, the linear sensitivity algorithm is applied to a modified IEEE 33-bus distribution system to demonstrate its extended effectiveness to voltage monitoring and control scenarios. Its application estimates and compensates in a better way the voltage deviations with regard to the operating desired voltage $\left(|V|_{o p}\right)$ constraints, using distributed power injections at the PCCs. Numerical results always showed a curtailment of the relative error against common simplifications of the electrical modelling in steady-state, thus simulating two critical scenarios of operation production-consumption for the existing system response. The proposed algorithm was validated considering as reference the voltage profile outputs of the load flow analysis, using the Newton-Raphson method via DIgSILENT, in terms of its accuracy, silhouette shape along the feeder and with regard to the application of reactive compensation as an analytical case study for voltage improvement in active distribution networks.
\end{abstract}

Keywords: DER operation; $V-Q$ regulation; sensitivity analysis; distributed power injections; distribution-system modelling; voltage profile monitoring; ancillary services DG; voltage improvement

\section{Introduction}

By 2050, renewable sources will account for $80 \%$ of total electricity generation [1], which presents certain challenges to system operators. In terms of power system modelling, existing downstream grids have not been structurally designed for the operation of distributed energy resources (DER). Despite this, identifying ancillary services provided by distributed generation (DG) to the electric power system (EPS) can be seen as an opportunity to create a framework for the operational flexibility of the network [2]. In fact, one of the most significant advantages of DER operating over power quality is the strategic improvement of voltage profiles [3].

In an analytical framework, prior to the implementation of an optimization scheme for operating DER, modelling and simulating should consider models for the monitoring of distributed power injections, easing their smooth integration into the new system-i.e., network-DER interoperability - and minimizing errors due to assumptions on mathematical models for the distribution system (DS). Therefore, estimating a state variable such as voltage based on the conditions of the existing network presents a problem related to the legitimacy of the assumptions made to simplify the model. 
In this paper, we propose an improved technique for analytical modelling of the voltage profile in distribution networks, which is based on a matrix of linear sensitivities of the power flow in a steady state, as a function of the topological characteristics of the network: the branch resistance $(R)$, branch inductive reactance $(X)$, the capacitive susceptance $\left(B_{c}\right)$ under radial operation, the load buses of active power $(P)$ and reactive power $(Q)$, and injections of distributed power along each feeder of the DS. The applied improvement includes a linearised formulation in function the variables shown above, which are well known in the literature, and considering the feeder capacitance into the modelling.

To achieve a comprehensive analysis, we focused on the simplest model of a feeder, as illustrated in Figure 1. In addition, a trend scheme of voltage profiles is shown, theoretically illustrating how the profile of the existing network could vary toward a new operating point $(P e, Q e)$ inside of controlled scenarios of power injection/absorption. Our focus in this paper concerns the network sensitivities at the points of common coupling (PCCs), as sources of collected power at the feeder. Active and reactive power consumption will result in a voltage drop along a medium-voltage (MV) feeder, which is the lowest at minimum consumption and highest at maximum consumption [4]. New operating conditions using DER for voltage regulation at PCC arise as requirements of a steady-state analysis that is dependent on the network sensitivity.

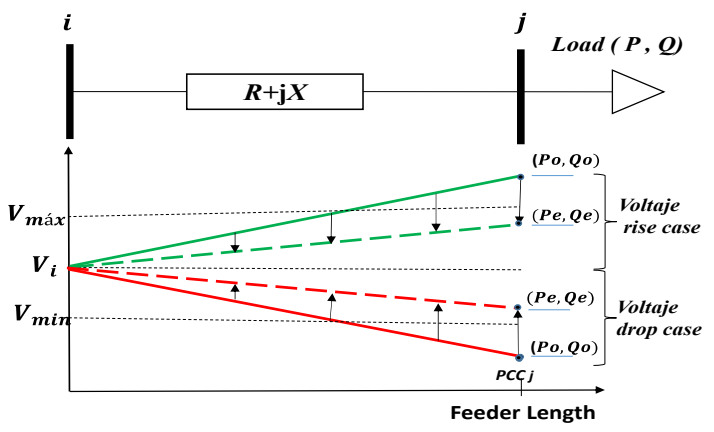

Note: (Po, Qo) corresponds to an initial operating point with regard to the consumption of active and reactive power in bus $j$, current load; $(P e, Q e)$ corresponds to a new operating point for the power balance at point of common coupling (PCC), adding power injection/absorption to the self-consumption bus. In this case, the selfconsumption bus is the same bus PCC interconnected to the distribution network.

Figure 1. Load feeder and trend of voltage profiles.

In the literature, the longitudinal voltage deviation (1) between two buses $i$ and $j$ of a feeder-and the derived expressions-is accepted as the traditional method for performing a sensitive analysis of the voltage profiles, including the topological structure of the network.

$$
\left|V_{i}\right|-\left|V_{j}\right|=\frac{R P+X Q}{V_{i}}
$$

This linear formulation can be improved through a direct mathematical algorithm, retaining the advantages of modelling and speed for subsequent optimisation studies. Researchers have applied this method in the analysis of the power hosting capacity of DER [5], estimation and voltage control in load nodes [6], and reactive power compensation [7,8]. Section 2 shows a mathematical model to enlarge the range of scenarios for new operating conditions with DER at PCCs.

However, the injection of distributed power into a network prompts variations in the load flow to each line-section with PCC. Currently, evidence shows the feasibility of regulating the voltage from DG. Nevertheless, as detailed by the Electric Power Research Institute (EPRI) in [9], in order to maintain the power quality, the amount of injected power from DER should not be arbitrary but should be monitored by distribution system operators (DSOs), in accordance with the characteristics of the existing network for the operational 
control schemes. In this way, it would be possible to mitigate the alerted impacts over the need for network operation, related to the criteria of new PCC locations [10].

Performance issues regarding voltage regulation can be mitigated via integrated voltage control and reactive power $(Q)$ management [11]. These solutions are classified into three types of method [12]: (i) reduction of the line impedance $Z=R+j X$; (ii) reduction of the generator power output, and (iii) increase of the generator reactive power import. Particularly, distribution networks have been modelled using representative networks and simplified models, depending on the scaling of parametric bounds and operating constraints with regard to the transmission system (TS) such as radial operation, $R / X$ ratio, lines features, and load sizes and type [13].

Currently, the operating analytical boundaries for TS can address the TS-DS interface and the existing distribution network up to MV levels with observability; therefore, analytical techniques for linear power flow can be used to monitor an acceptable and controllable voltage profile for standard operation using DER [14]. In fact, DG shares candidate locations for the capacitor banks placement, as both are dependent on the loss sensitivity factors and network configuration $[15,16]$ and operating point $P-Q$ values to satisfy the mandatory needs of industrial customers with large inductive loads. In this case, complementarily, the injected reactive compensation from DG can support voltage regulation.

Dealing with the import of $Q$ as a special case, in [17], different voltage profiles were analysed depending on the load profiles (industrial, residential, and commercial) with progressive penetration of the reactive power, quantifying fluctuations, and voltage variations. In understanding the $Q-V$ coordination for a control approach, the analytical model with DG must involve two power capacity boundaries: first, the $Q$-injection capacity according to the generation output from DG technology (for instance, asynchronous generators, such as wind turbines [18,19] and solar photovoltaic plant inverters [20]), and second, a radial feeder connected at the PCC (or a set of feeders of the system under study) engaging enough power transfer capacity [21], which is related to the design parameters and analytical modelling for the hosting capacity.

Hence, variations in the bus voltages and branch flows due to the injection of active/reactive power to whichever bus depend on the network topology [22]. The accuracy of the modelling increases when the radial configuration of the distribution network is considered, which is a property of the linear sensitivity as a function of the voltage drop [13], which in turn results from the initial load flow before linear transformation. The analytical procedure of direct branch flow presented here facilitates the conditions for the estimation of an optimal power flow; thus, the present work addresses the analytical solution of the problem from the modelling of the operating constraint, since nonlinear and optimised solutions [23-25] do not consider the condition of shared self-consumption, where examination of the sensitivity of the voltage profile by the influenced topological zones along the feeder is required. Due to the use of the matrix scheme of a direct load flow based on [26], the proposed method has a computational advantage and, thus, does not use the sensitivity matrix extracted from the Jacobian, which requires inversion [27].

There are still power dispatch scenarios not contemplated by DSOs in relation to the traditional DS operation, due to factors such as the lack of observability (measurement not available on the existing network), technical thresholds for equipment, and market constraints. However, their technical development is analytically feasible for new ancillary services under secure conditions, such as voltage control support, spinning reserves, load following, and peak shaving, among others [28]. Therefore, one motivation of our current paper is to present a linear sensitivity algorithm for the computing of voltage profiles and feasible operational variations, to obtain better voltage approximations for distributed power injections events $(P, Q)$ at PCCs along an existing distribution feeder. This accuracy is then exploited to monitor a case study by applying reactive compensation Q-V from DG and variations on the feeder loadability. 
This paper is organised as follows. Section 2 starts with a mathematical procedure for finding voltage deviations using power injections at the PCC in the feeder model. Section 3 explains the proposed method using a linear technique of voltage sensitivity in Section 3.1, and describing a geometric analysis of the full formulation of voltage deviation between two nodes and the forming of sensitivity matrices in Section 3.2. Section 4 provides the simulation of case studies, and critical production-consumption scenarios are presented to test the sensitivity algorithm for the voltage operating conditions. The results, including voltage profiles along the feeder and relative errors, are presented and discussed in Section 5. Finally, Section 6 draws our main conclusions regarding the proposed algorithm and outcomes in respect of its applicability.

\section{Mathematical Model of Voltage Deviations and P-Q Injections}

Considering a simple-DS through its single-phase equivalent between two buses, the power flow setting for voltage regulation can be illustrated as in Figure 2 [29].

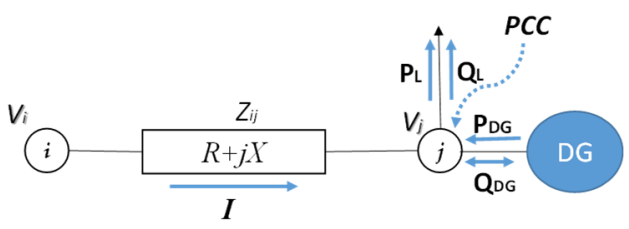

Figure 2. A two-bus simple distribution system with distributed generation (DG).

In rectangular form, an apparent power flow $(S)$ is calculated, as shown in Equation (2):

$$
S=P+j Q=\left(P_{L}-P_{D G}\right)+j\left(Q_{L} \mp Q_{D G}\right)
$$

where $S$ represents apparent power that flows between $i$ and $j$ on the line impedance $Z_{i j}$, and the subscripts are related to the power consumption and production, i.e., load $(L)$ and distributed generation $(D G)$, respectively. The bus $j$ represents a point of common coupling (PCC) for DG with self-consumption, and bus $i$ corresponds to the slack bus over the substation with the voltage $1.0 \mathrm{pu}$.

Applying Kirchhoff's current law (KCL), we deduced from the mathematical model of the branch circuit

$$
\hat{V}_{j}=\hat{V}_{i}-\hat{I} Z_{i j}=\hat{V}_{i}-\hat{I}(R+j X)
$$

and

$$
\hat{I}=\frac{P-j Q}{\hat{V}_{i}^{*}}
$$

Therefore, replacing Equation (4) in Equation (3), the expression for voltage drop is given as:

$$
\Delta \hat{V}_{i j}=\hat{V}_{i}-\hat{V}_{j}=\frac{R P+X Q}{\hat{V}_{i}^{*}}+\mathrm{j} \frac{X P-R Q}{\hat{V}_{i}^{*}}
$$

The complex expression for voltage deviation in Equation (5) is simplified and bounded to its real component for the traditional method. Several authors have studied voltage control using sensitivity analysis for certain applications of the operational stage, i.e., using a load control technique [12], analysis based on the worst-case scenario of distribution network for the voltage control approach through reactive power compensation [7], reactive power with particle swarm optimization (PSO) algorithm [8], or direct sensitivity analysis in MV networks [30,31].

F3or voltage deviation conditions, the $X / R$ ratio is quite sensitive to transfer power in transmission branches, where $X$ is higher. Conversely, for distribution systems, the $R / X$ ratio is significant, being increasingly resistive. For levels of medium voltage (MV), the imaginary part of Equation (5) is also neglected because the voltage angle between 
the buses $i$ and $j$ is relatively small. However, the term $R Q$ is analytically relevant for DER-operation through the sensitive effect along the voltage profile.

Due to the aforementioned interoperability, the analysed scenario with selfconsumption and $Q$-compensation modifies the constraints under consideration, depending on the access point at the DS feeders and sub-transmission level networks [32]. Then, the sensitive voltage assessment approach implies feasible sources of injection or consumption on active buses to bring forward this required adjustment for compensation. Among other things, this achieves an accurate voltage magnitude calculation based on the matrix method of linear sensitivity, and the direct branch flows are affected by the $P-Q$ injections.

Both components (real and imaginary) of Equation (5) relate the power flow over the topological characteristic $R / X$, which is not negligible in distribution systems (typically $\geq 1$ ). Below is the $\pi$-model of a distribution line with PCC in Figure 3.

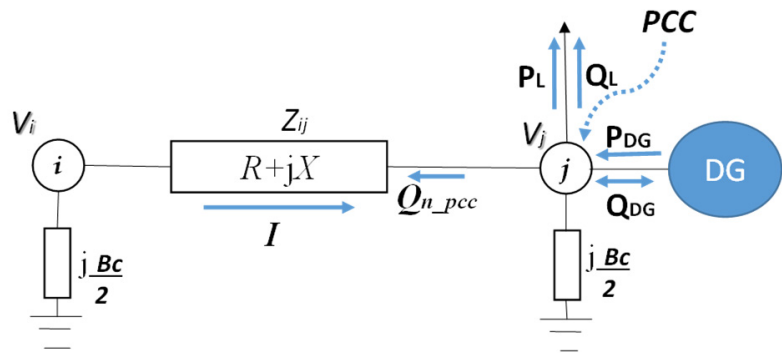

Figure 3. A proposed $\pi$-model of power line with point of common coupling (PCC).

Although the classic $\pi$-model assumes current divisions toward the capacitive susceptances, in the case of the radial operation, this model adds half of the capacitive susceptance involved in each node that shapes each line segment. In this way, the total expression for the net reactive power at PCC is:

$$
Q_{n_{-} p c c}=\left(-Q_{L} \mp Q_{D G}\right)+Q_{B c}
$$

where the $Q \operatorname{load}\left(Q_{L}\right)$ is a consumption sink with a negative sign (-) in Equation (6); DG power: in case $Q_{D G}$, inductive with a negative sign (-); $Q_{D G}$ capacitive with a positive sign (+); and the reactive power due to the line capacitive susceptance added, given by $Q_{B c}=\frac{B_{c}}{2}\left|V_{j}\right|^{2}$. Here, the generator sign convention is used. This means that when the reactive power is positive, the component shows capacitive behaviour and when $Q$ is negative, the component shows inductive behaviour.

Accordingly, the line shunt capacitance feeds the reactive power of the system [33]. In this way, the voltage error relative to the analytical model can be reduced for feeder monitoring. The sensitive effect of $Q$-injections on the feeder voltage is reinforced longitudinally by integrating the capacitive susceptances at the tie DG-PCC. This application highlights the relevance of the RQ term in DS analysis. Such a contribution to the $V$ - $Q$ compensation is reproduced along the matrix of the sensitivity of the feeder. Ideally, considering the possibility of a network where all nodes are DG-PCC, this effect would be quantified on the voltage profile in the same way. The effect would increase notably at nodes with a greater number of branches connected at PCC and nodes further away from the slack bus.

Consequently, for the presented modelling, our interest is in the total load flow through the branch between the buses $i$ and $j$, based on a direct model of current injections to the bus (BIBC: bus-injection to branch-current), according to [26]. For the voltage control at the ends of the feeder, $V_{i}$ and $V_{j}$ are mutually dependent voltages. When the generators are connected to the feeder, the voltage profile is likely to increase [34] compared to the base scenario according to the voltage sensitivity at the self-consumption bus.

Given that any variation of $P$ or $Q$ is significant in a network with high voltage sensitivity, as is the case for the distribution system in this study, the sensitive effect of 
power variation over voltage modules was analytically maintained through the topological sensitivity along the feeder.

In the following, in Figure 4, a schematic guide of the modelling solution is presented:

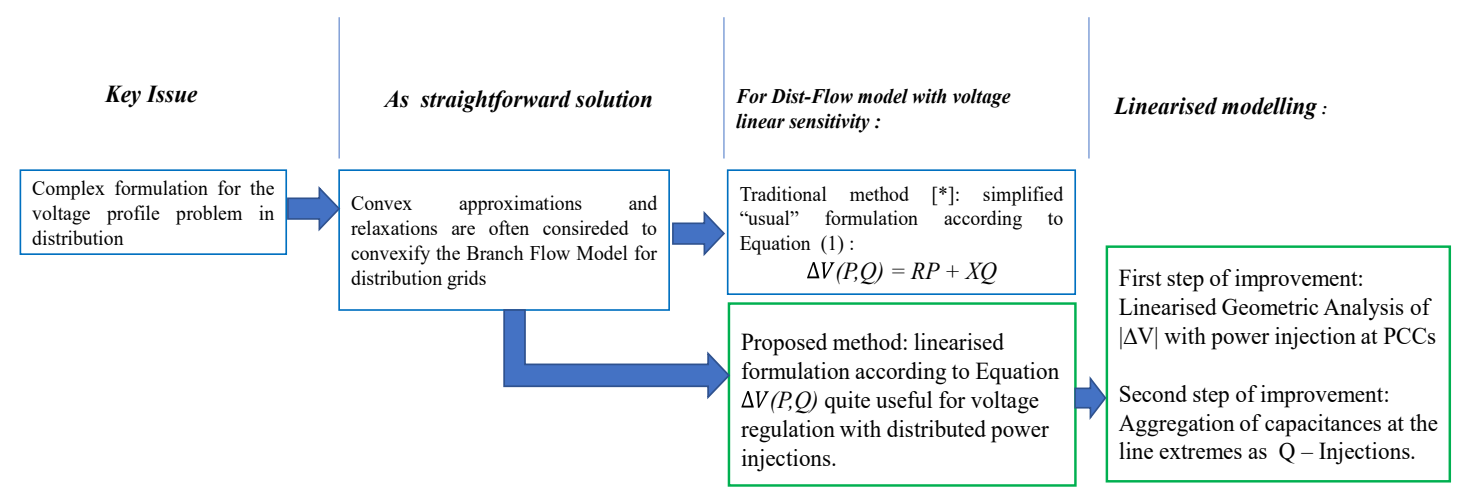

Figure 4. Guide scheme for the modelling solution. Note: [] Recent studies [35-38].

\section{Proposed Method}

The following is a brief conceptualization of the sensitivity analysis: a methodological articulation of the voltage sensitivity with regard to the topology, the mathematical formulation, and its application to the system.

\subsection{Description of the Linear Sensitivity of the Voltage at the PCC}

Generally, the sensitivity of control variables is considered to be inversely proportional to the robustness of the system. In power systems, it is usual to analyse the sensitivity of an electrical variable, such as the voltage in the bus $j$, with respect to another variable, such as the reactive power [39]. The difference lies in the analytical approach that is followed in order to establish control guidelines between both variables.

From the approach of power injection, the sensitive effect on the voltage deviation is fulfilled considering suitably the following function for each node with self-consumption:

$$
\Delta V=F_{P}(\Delta P)+F_{Q}(\Delta Q)
$$

where

$$
\begin{gathered}
\Delta P=P_{o}-P_{e} \\
\Delta Q=Q_{o}-Q_{e}
\end{gathered}
$$

Equation (7) is a sensitivity function $(F)$. These implicit functions $F_{P}$ and $F_{Q}$ quantify the voltage-module contribution at the PCC due to $P$ and $Q$, respectively. In this case, the initial operating point of the net power $\left(P_{o}, Q_{o}\right)$ changes to reach a desired voltage value in each bus. $P_{e}$ and $Q_{e}$ correspond to the end powers after variation due to power injections at the PCCs. This condition denotes nodal compensation in order to manage the operating voltage limit along the feeder.

For the simulation, two sources of information were established as input data: system topology data and a load flow of the initial conditions to identify the base steady-state scenario of the existing network. This technique can be used to calculate voltage variations around the network with respect to the slack bus. The voltage variations due to $Q$ injection are performed from the power factor control at the shared self-consumption bus.

Functionally, as a preliminary step for the mathematical optimization of objective functions, the application of sensitivity theory to the injection problem, for either direct dispatch analysis or monitoring and metering, allows a considerable simplification of the computational difficulties encountered in control theory. The aim of the sensitivity analysis is to estimate the variation of the power flow with regard to feasible small changes in the state parameters, affecting new proposals and the analytical model of the existing network. 
Something important about this sensitivity analysis is that the treatment of the linearised formulation does not increase the computational burden.

Considering the integrated variables for the proposed model in Figure 3, with its implicit variables to the vectorial equation, a small geometry can be configured to analyse a complete voltage drop $\Delta V$ in looking for a linear expression.

Linearised Geometric Analysis of $|\Delta V|$ :

In the following diagram (see Figure 5), through a geometric analysis, an analytical expression for the voltage drop $\Delta V$ is found, in function of the power injection and nodal consumption at the PCC, line impedances, and difference between voltage angles.

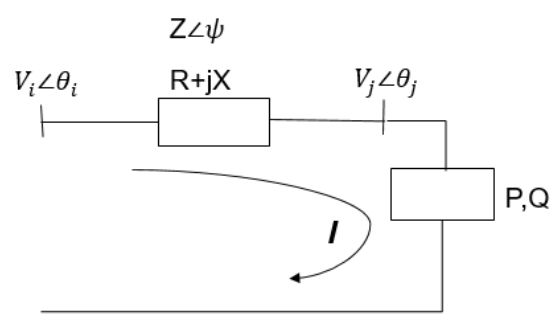

(a)

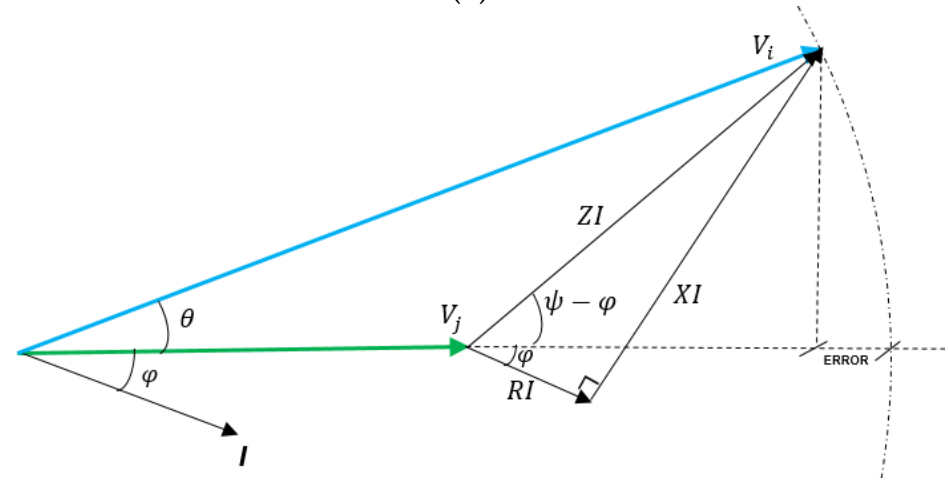

(b)

Here $\varphi$ is the current angle and, in this case, the load power factor angle; $\psi$ is the line impedance angle; and $\theta=\theta_{i}-\theta_{j}$ is the voltage angle between buses $i$ and $j$.

Figure 5. (a) Single-phase scheme. (b) Phasor diagram.

In Figure $5 b$ is indicated a potential error in the voltage deviation magnitude.

According to Figure 6, by rotating the voltage modulus, $|V i|$ about $|V j|$, a complete voltage deviation is identified, taking into account $P, Q, X, R$, and the resulting relationships from the direct load flow between two nodes for such variables.

$\mathrm{O}, \mathrm{A}$, and B in Figure 6 enclose this exercise on the voltage modules of $|\mathrm{Vi}|$ and $|\mathrm{Vj}|$. The illustrated case shows a voltage drop; in the same way this can be done for a voltage rise $(V j>V i)$. In this portion of the circumference, the radius corresponds to the voltage module.

In Figure 6, the segments $\overline{D^{\prime} B}$ and $\overline{D^{\prime} D}$ can be calculated as follows:

$$
\overline{D^{\prime} B}=\left(V_{i}-V_{j}\right) \sin \left(\frac{\theta}{2}\right)
$$

and

$$
\overline{D \prime D}=\left(V_{i}-V_{j}\right) * \cos \left(\frac{\theta}{2}\right)=I * Z * \cos \left(\psi-\varphi-\frac{\theta}{2}\right)
$$




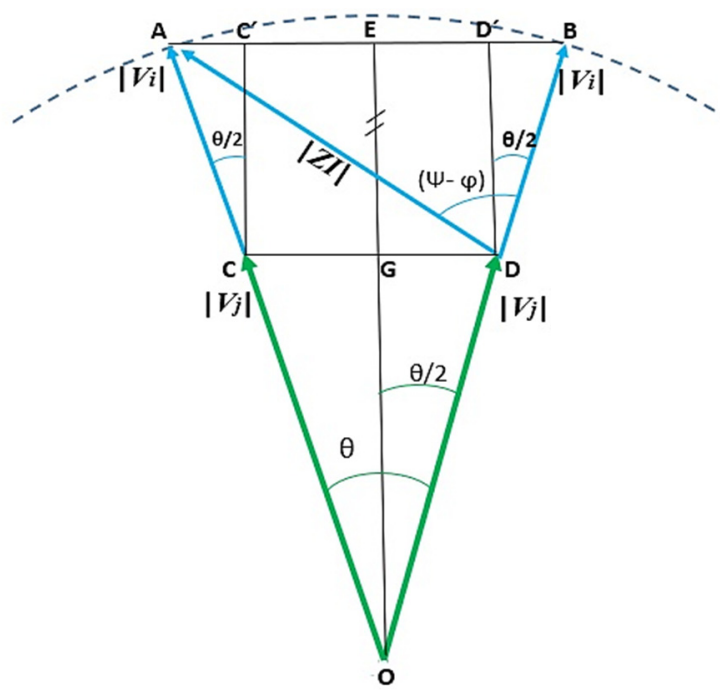

Figure 6. Voltage magnitude diagram between two nodes.

In the same way, geometrically, it is satisfied that:

$$
\left(V_{i}-V_{j}\right)^{2}=\left(V_{i}-V_{j}\right)^{2} * \sin ^{2}\left(\frac{\theta}{2}\right)+(I * Z)^{2} * \cos ^{2}\left(\psi-\varphi-\frac{\theta}{2}\right)
$$

It is necessary to use the following trigonometric identities (13), (14), and (15):

$$
\begin{gathered}
\cos ^{2}\left(\frac{\theta}{2}\right)=1-\sin ^{2}\left(\frac{\theta}{2}\right) \\
\cos (\psi-\varphi)=\cos (\psi) \cos (\varphi)+\operatorname{sen}(\psi) \operatorname{sen}(\varphi) \\
\operatorname{sen}(\psi-\varphi)=\operatorname{sen}(\psi) \cos (\varphi)-\cos (\psi) \operatorname{sen}(\varphi) .
\end{gathered}
$$

Using Equation (13), expression (12) can be written as:

$$
\left(V_{i}-V_{j}\right) \cos \left(\frac{\theta}{2}\right)=I * Z \cos \left(\psi-\varphi-\frac{\theta}{2}\right)=I * Z\left[\cos (\psi-\varphi) \cos \left(\frac{\theta}{2}\right)+\sin (\psi-\varphi) \sin \left(\frac{\theta}{2}\right)\right]
$$

On each PCC in the feeder, the power triangle relationships are given by $S^{*} \cos (\varphi)=P$ and $S^{*} \sin (\varphi)=Q$ for the active and reactive powers, respectively. Similarly, the magnitude of line parameters $R$ and $X$, is given by $Z^{*} \cos (\psi)=R$ and $Z^{*} \sin (\psi)=X$, respectively, according to the impedance triangle.

By replacing the power and impedance expressions in Equation (16), using (14) and (15), the full expression for the voltage deviation magnitude can be obtained:

$$
\left(V_{i}-V_{j}\right)=\frac{[R * P+X * Q]+\tan \left(\frac{\theta}{2}\right)[X * P-R * Q]}{V_{i}}
$$

In Equation (17), a new resulting unknown variable is introduced, its value relating to the voltage angle difference in this analysis: $\tan \left(\frac{\theta}{2}\right)$, which contributes to the amplitude of the voltage drop up to the analysed node.

The analytical parameter $\tan \left(\frac{\theta}{2}\right)$ can be reviewed more accurately in future studies for the optimization of the voltage profile. However, in order to consider it here, it is useful 
to use an approximation resulting from the quotient between $\frac{P X-Q R}{V_{i}}$ and $V_{i}$ in terms of the known components $(P, Q, X, R$, and $V)$ for nominal voltage values as is shown by:

$$
\tan \left(\frac{\theta}{2}\right) \cong \frac{P X-Q R}{V_{i}^{2}}
$$

Therefore, by replacing (18) in the formulation (17), the complete expression for a voltage deviation would be given as:

$$
\Delta V_{i j}=\frac{[R * P+X * Q]}{V_{i}}+\frac{[X * P-R * Q]^{2}}{V_{i}^{3}}
$$

Thus, a second additional term is evident for expression (1), which is non-significant for very high voltage levels, as in transmission networks, or when $X P \cong R Q$. In other words, it is important for sub-transmission levels and DSs.

The sum of the impedances from the slack bus is used to build a complete voltage profile. The uncompensated value of power $(P, Q)$ is necessary as an input condition in the proposed algorithm. As the calculation is direct, it is feasible to quantify the effect of the sensitivity on the voltage variation through the matrix equation with known input constants from an initial power flow.

\subsection{Forming a Sensitivity Matrix to [R] and [X]}

To illustrate the matrix construction of the direct power flow for the voltage sensitivity analysis, a simple diagram of four buses is shown in Figure 7:

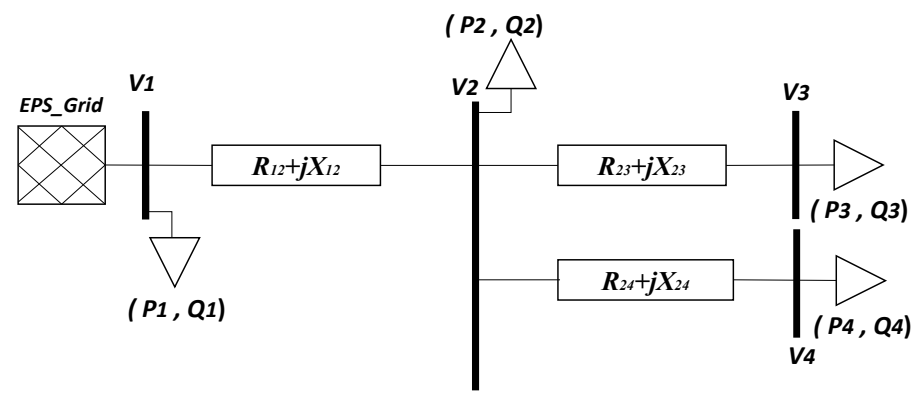

Figure 7. A simple four-bus radial distribution system.

A set of voltage deviations along the network can be obtained with the partitioned matrix formulation built based on bus injections for a simplified branch power flow, as follows from Equation (19):

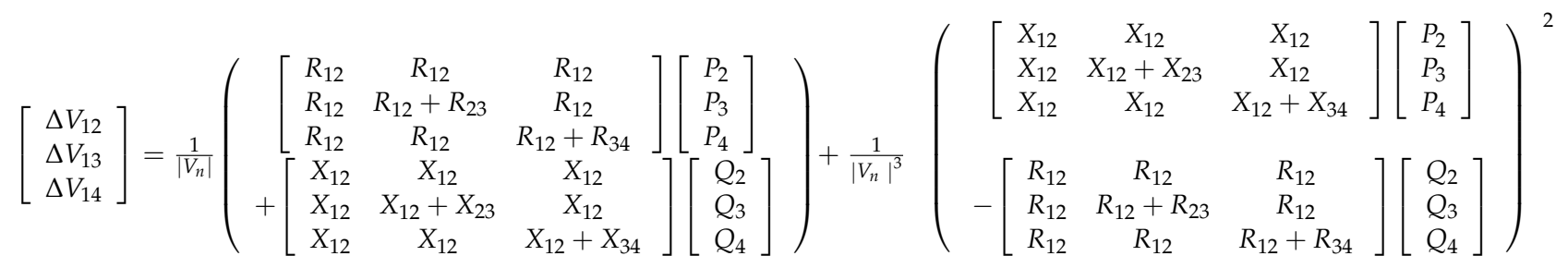

where $|V n|=$ the nominal voltage of the network (1.0 pu).

The contribution from the slack $(i=1)$ bus by $[P]_{3 \times 1}$ and $[Q]_{3 x 1}$ injection to the load at buses 2, 3, and 4, contains a voltage difference along the radial path. In Equation (20), the matrices $[R]_{3 \times 3}$ and $[X]_{3 \times 3}$ represent voltage sensitivity matrices with regard to the power injections or load requirements. 
As the advantage of matrix forming for the existing network is done only once for all simulation scenarios, any changes in the feeder line segments can be modified in the current layout.

\section{Simulation: Case Study Test}

\subsection{Case Study: 33-Bus Modified Network for DG}

To validate the analytical procedure, the 33-bus reference system shown in Figure 8 was used, with the rated load and topology information that appears in [40]. This is a medium-voltage network $(12.66 \mathrm{kV})$, consisting of four PCCs located along the feeder, on buses $6,12,18$, and 33. The DG units are DFIG-WT (wind turbine) type, and there is a nominal power of 1 MVA for each of them.

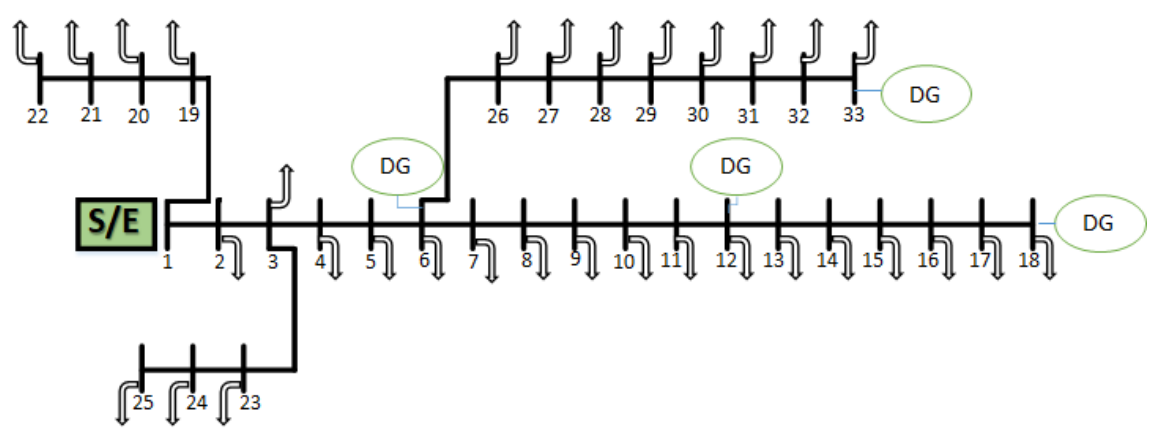

Figure 8. A 33-bus radial distribution system.

The network has three feeder zones: the feeder head at segments 1-6, 2-22, and 3-25; the intermediate zone at bifurcations 6-33 and 6-12; and the extreme zone of the feeder at the segment lines between buses 13-18. For the present work, the tie lines from the original network were not included.

Retaining the proposed $\pi$-model in Figure 3, the effect of the capacitive susceptance for the voltage sensitivity increases due to the line length, insulation material (overhead or underground lines), and number of branches connected to each bus.

The versatility of the developed matrix algorithm allows such an effect to be quantified as this capacity is contemplated. In the case of overhead lines, the shunt susceptance $\left(B_{C}\right)$ is typically lower. For underground lines, $B_{C}$ has values from $\approx 0.217 \mu \mathrm{F} / \mathrm{km}$ to $0.302 \mu \mathrm{F} / \mathrm{km}$, which is a function of the insulation material and diameters for $\mathrm{MV}$ conductors less than $36 \mathrm{kV}$. To show the outcome, a scenario with a high reactive load was assessed and is demonstrated by the evidence presented here.

\subsection{Parameter Settings for Operating Scenarios: Consumption-Production}

To test the existing network sensitivity outlined in Section 3, two scenarios of critical impact on voltage variation with DG were selected, a consumption scenario (CS) and a production scenario (PS). The CS scenario presents the maximum nominal load of the system with a scale factor of 1.0 and the minimum generation injected at PCC with a rated power scale factor of 0.15 . The PS scenario consists of the minimum load with a scale factor of 0.25 and generation with a maximum scale factor of 0.9 . These cases are realistic test scenarios, in which $Q$ injection is performed, in order to return to the allowed operating voltage limits.

The present technique allows the use of the most sensitive point of the network to inject power. In the case of the power factor variation, the required $Q$ at the self-consumptions buses holds the capacity curve of each generating unit.

\subsection{Determination of Operating Point DG}

As a criterion for establishing the capacity of the power curve of the DG units, which is a DFIG-WT type with a maximum power of $1 \mathrm{MVA}$, the power injection thresholds for 
$P_{D G}$ and $Q_{D G}$ are taken into account [19], as per the following four operating points with a nominal voltage in Table 1.

Table 1. The operating points of DFIG-WT. Rated injection capability of reactive power (Qinj) versus active power (Pinj).

\begin{tabular}{cccc}
\hline Operation Point & Pinj (\%) & Qinj (\%) & Power Factor \\
\hline I & 0 & \pm 0.95 & 0 \\
II & 0.25 & \pm 0.95 & 0.25449 \\
III & 0.5 & \pm 0.90 & 0.485642 \\
IV & 1 & \pm 0.6 & 0.857492 \\
\hline
\end{tabular}

During the power delivery from the distributed units, each discrete value of injected reactive power $\left(Q_{i n j}\right)$ versus injected active power $\left(P_{i n j}\right)$ with nominal voltage is the minimum point for the operation interval of the generator curve. These values are chosen as feasible reference for the operational range. At the point of the greatest sensitivity of the voltage to the injection of $Q$, a minimum $Q$ was injected according to the requirements of the self-consumption scenario. Depending on the load level $(P, Q)$ in each node, it was possible that nodes with the highest voltage drop did not necessarily match the PCC buses, and it was necessary to compensate $Q$ from neighbouring nodes.

According to the preliminary results, we confirmed that the zone closer to the substation had almost no voltage variability, which was also found in branches without PCCs in contrast to buses next to power injections. Similarly, there were nodes that were not affected by the injected generation, which also remained relatively constant. The effect was predictable for the intermediate levels with the simultaneous connection of DGs; hence, the complete analysis started from a constant, which was the sensitivity of the topological matrix of the system. In other words, the effect of the voltage sensitivity, which increases along the topological matrix, is constant from the design memory of the existing system. The topological matrix is built only once and it will be modified when the DSO adds or removes matrix lines, in the case of circuit expansion or network reconfigurations, respectively.

\subsection{Operating Constraint}

For the voltage profile, it is necessary to monitor its operating constraint margins (network with " $\mathrm{m}$ " buses):

$$
V_{\min }<|V|_{o p}<V_{\max }
$$

where

$$
|V|_{\mathrm{op}}=\left[\begin{array}{c}
V_{\text {slack }} \\
V_{1} \\
V_{2} \\
\vdots \\
V_{m}
\end{array}\right]_{O P} \in(\text { Vmin., Vmax. })
$$

where $V_{\text {min }}$ and $V_{\text {max }}$ are the lower and upper bounds of the bus voltage limits, respectively, and $|V|_{o p}$ is the operating voltage magnitude. According to the new IEEE Standard 1547-2018, as the requirements of power quality are mandatory on the MV level, there is an allowable voltage deviation of $\pm 3 \%$ of the rated value for each bus for regulating the interoperability with DER.

\subsection{Simulation Conditions}

To define a required operating point that is feasible for the voltage profile, we established a flowchart (see Figure 9) that generates varied cases in a controlled manner. The power factor of the self-consumed nominal load is subject to discrete variations for the applied sensitivity analysis. For any base case, there are two types of results, with and 
without $Q$ injection, with regard to $P$ injection, as shown in Table 1 . It is important to define the boundaries for a feasible $Q$ injection interval.

$$
\Delta F_{d s}=\frac{\text { Upper limit }- \text { Lower limit }}{k}
$$

where $\Delta F_{d s}$ is a discrete incremental step that depends on the sensitive shift parameter. For example, if the range is from 0 to $1 \mathrm{pu}$, the discrete step is 0.05 for $k=20$.

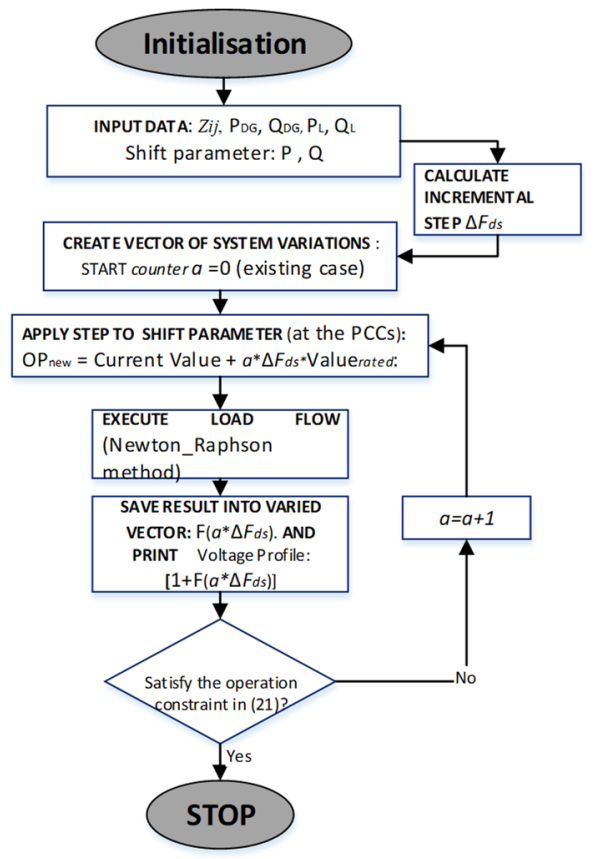

Figure 9. Flowchart to create initial conditions of the operating points.

The linear sensitivity modelling is a result of controlled variations of the shift parameter to reach an operating point that retains the operating constraints for the network, and, at the same time, must satisfy the injection curve of each generating unit.

Hence, the voltage profile at each point of the network is guaranteed to quantify the rapid variations due to the penetration of DER, within standard limits, and the relative error is reduced with the algorithm presented and validated against the Newton-Raphson method (N-R) by the current equations for distribution systems.

In this paper $\Delta F_{d s}$ is considered as $0.05 \mathrm{pu}$ for calculating the simulated sensitivity scenarios.

In this way, the operating points for the control injections were obtained, and the relative error with respect to linear sensitivity analysis was determined. These results serve as input data for the diagram in Figure 10:

The relative error was calculated in the following way, comparing the resulting value in the N-R Load Flow $(L F)$ with the value found by the proposed algorithm:

$$
\varepsilon=\frac{\text { value } L F-\text { value proposed method }}{\text { value } L F} * 100 \%
$$

The sensitivity analysis was applied for small deviations or perturbations in the steady-state analysis, that is, when it was necessary to bring the voltage profile to the limits of normal operation in order to inject small amounts of power to feeder segments with greater sensitivity. It is not applicable for voltage restoration with frequency stability management or voltage collapses. 


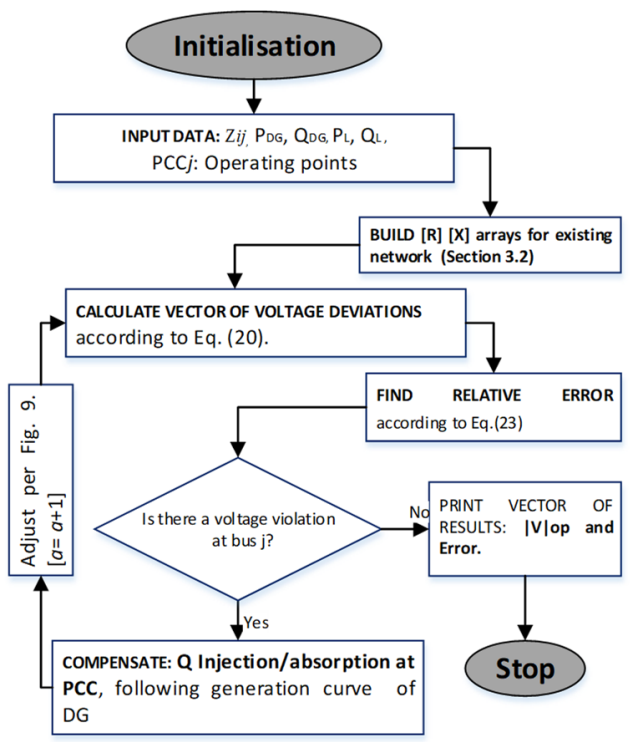

Figure 10. Algorithm flowchart of the control V-Q using linear sensitivity analysis at the PCCs.

\section{Simulation Results and Analysis}

In this section, several simulation results are reported for the application: a comparison of the performance against relative error, the response qualities in special cases of reactive compensation for critical consumption-generation scenarios, and a scenario with a high reactive load in the extreme zone of the feeder. The proposed algorithm was validated in DIgSILENT PowerFactory using the Newton-Raphson method. We applied the method to the 33-bus distribution system shown in Figure 8 to provide a comprehensive performance comparison.

\subsection{Performance Comparison: Silhouette and Accuracy}

Figure 11 shows the results of the sensitivity analysis for the proposed matrix model after simulating a consumption scenario. For this case, a maximum capacity of $0.302 \mu \mathrm{F} / \mathrm{km}$ was used, obtaining the following voltage profiles.

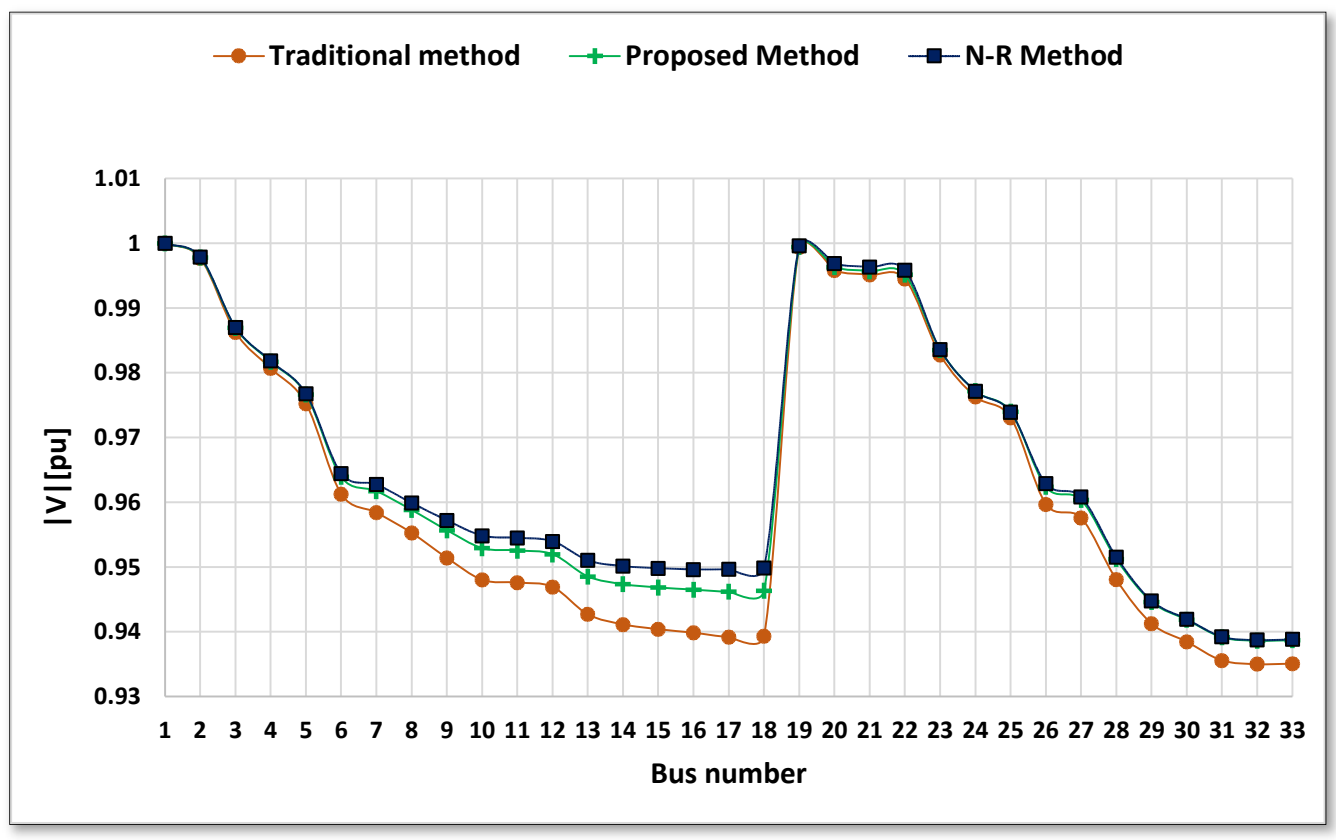

Figure 11. The voltage profiles for the 33-bus test system. 
Regarding the accuracy of the profile silhouette, as can be seen, when simulating only the conditions of a nominal load on the system, the proposed method demonstrated a better approximation to the voltage profile output via the N-R method. The PCC were buses $6,12,18$, and 33 .

In Figure 12, a reduction of the relative error was noted over the voltage profile in all buses, and was greater for the more distant points, PCCs 33 and 18. The proposed algorithm maintained a less steep relative error slope compared with the traditional method at load buses 2 to 18 . At bus $30(P=200 \mathrm{~kW}$ and $Q=600 \mathrm{kVAr})$, the reactive load was three times the magnitude of the power consumption $P$, and the error was mitigated in that circuit branch.

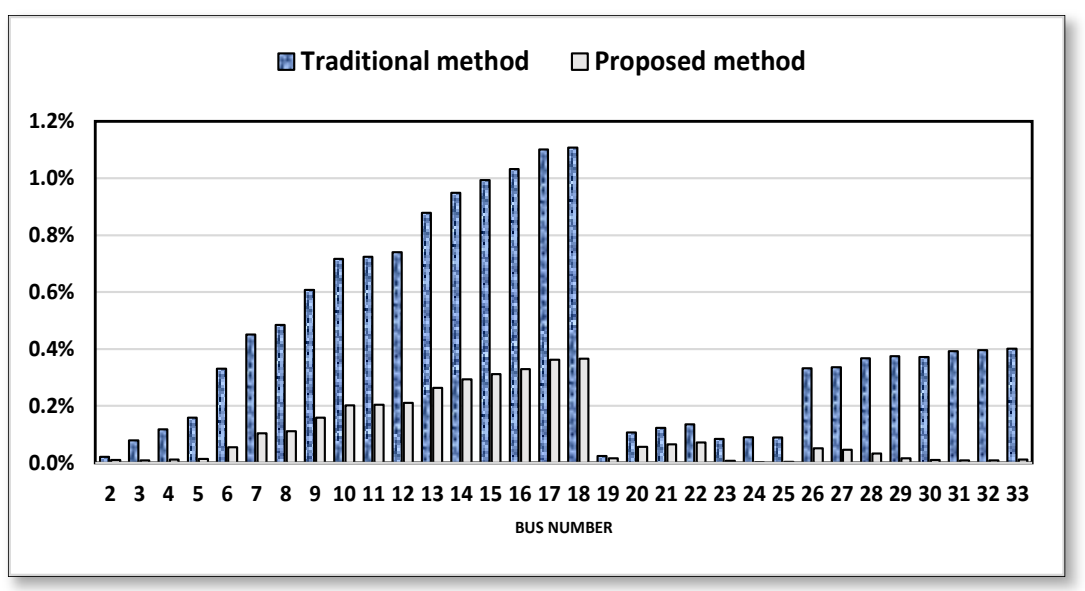

Figure 12. Relative error by sensitivity analysis.

The graph shows that, for node 33, the relative error became practically zero when using the proposed algorithm.

\subsection{Compensation $V-Q$ from the PCC $j$}

The generation-load conditions are given in Section 4.2. Figures 13 and 14 show the results of the proposed method for voltage profiles, applying compensation by the injection of $Q$ at the PCC of the system for the two operating scenarios illustrated (blue indicates scenarios with no compensation).

As shown in Figure 13, we analysed the voltage variation $(\Delta V)$ behaviour, applying the $Q$ injection at the PCC. For the CS, the voltage profile was operationally compensated with an injection of $750 \mathrm{kVAr}(+)$. In the PS scenario (see Figure 14), the absorption was 600 kVAr $(-)$, based on the scenario of the existing network. These injection values comply with realistic operating parameters for critical cases.

For both CS and PS scenarios, the bus voltages 19-22 remained relatively constant, which shows the absence of connected DG in the branches. We observed that, for a case without compensation, the traditional method and the proposed method had approximately the same precision and overlapped with each other.

Two relevant outcomes were identified with regard to the application of the proposed algorithm: an improvement in the effectiveness when there is a $Q$ injection for voltage compensation, and while a PCC is further away with respect to the slack bus, the error decreases. The latter can be seen for nodes 33 and 18, where the proposed method and the Newton-Raphson method were closer to each other than the traditional method was for the same compensation scenario.

For the compensated PS case, analytically, the traditional method still did not reach the maximum operational voltage limit, and this indicates greater absorption of $Q$. 


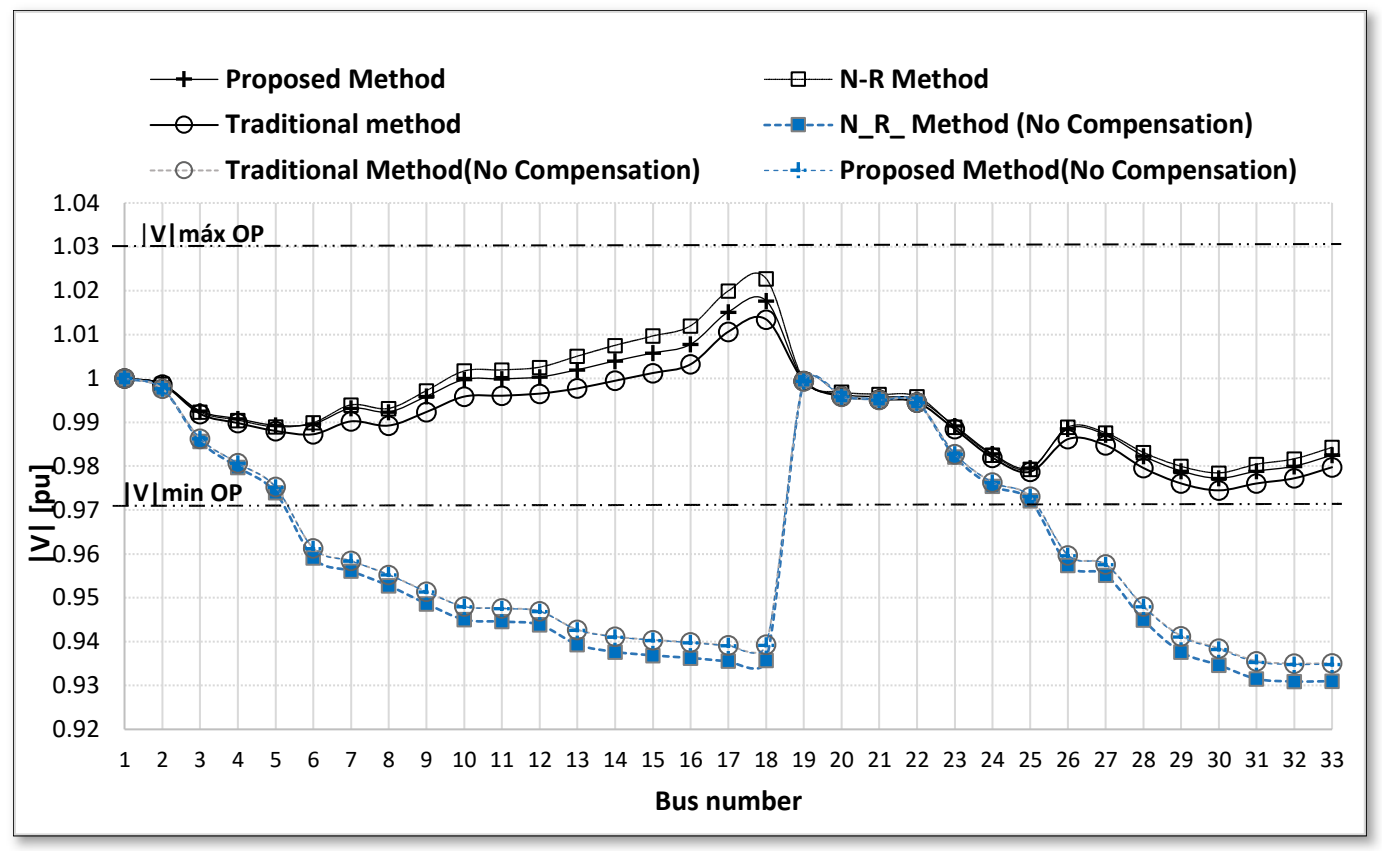

Figure 13. Consumption scenario (CS) voltage profiles. Compensation $V-Q$ case.

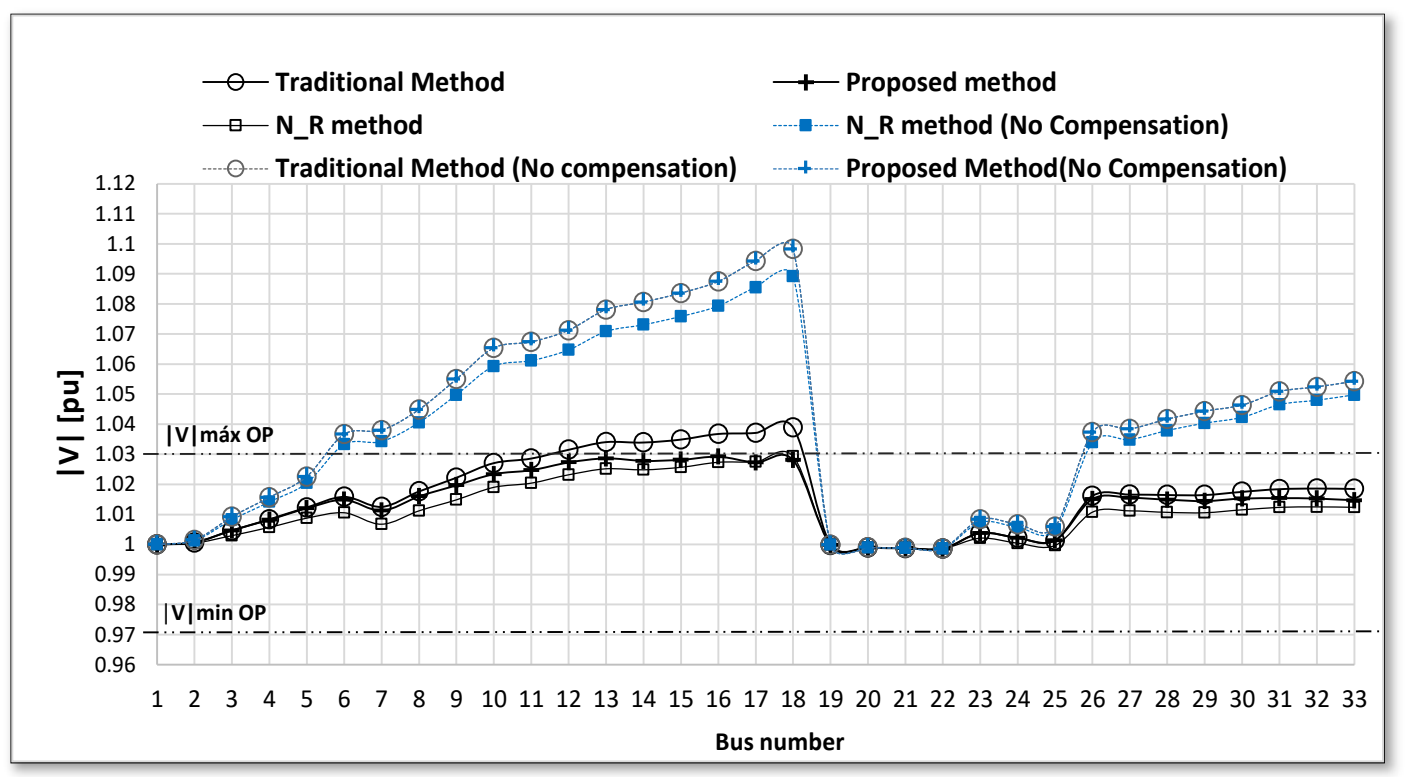

Figure 14. Production scenario (PS) voltage profiles. Compensation $V$ - $Q$ case.

The relative error from the sensitivity analysis is shown in Tables 2 and 3. For the CS scenario, the maximum relative errors for the voltage profile were $0.91 \%$ for the traditional method and $0.495 \%$ for the proposed method at the same PCC18, a difference of $0.415 \%$. This bus is further from the slack bus (approx. $24 \mathrm{~km}$ ). In the PS, the reduction value reached a difference of $0.818 \%$, which better validated the performance for the power injection.

With the exception of PCC6, which is located $3.6 \mathrm{~km}$ from the substation (the closest PCC bus to the substation), the proposed algorithm gave the best response for the production scenario of the PCC buses furthest from the substation rather than for the consumption scenario. This is consistent with the matrix sensitivity in these network zones of the network. 
Table 2. Relative error for the PCC-voltage in consumption scenario (CS).

\begin{tabular}{cccc}
\hline CS & Traditional Method & Proposed Method & Absolute Difference \\
\hline PCC & Rel. Error & Rel. Error & $\Delta \varepsilon$ \\
6 & $0.262 \%$ & $0.026 \%$ & $0.236 \%$ \\
12 & $0.598 \%$ & $0.221 \%$ & $0.377 \%$ \\
33 & $0.457 \%$ & $0.181 \%$ & $0.276 \%$ \\
18 & $0.910 \%$ & $0.495 \%$ & $0.415 \%$ \\
\hline
\end{tabular}

Table 3. Relative error for the PCC-voltage in production scenario (PS).

\begin{tabular}{cccc}
\hline PS & Traditional Method & Proposed Method & Absolute Difference \\
\hline PCC & Rel. Error & Rel. Error & $\Delta \varepsilon$ \\
6 & $0.526 \%$ & $0.427 \%$ & $0.099 \%$ \\
12 & $0.815 \%$ & $0.401 \%$ & $0.414 \%$ \\
33 & $0.604 \%$ & $0.230 \%$ & $0.374 \%$ \\
18 & $0.937 \%$ & $0.119 \%$ & $0.818 \%$ \\
\hline
\end{tabular}

A consumption scenario is shown below to demonstrate the effect of the shunt susceptance (only) without compensation, within the electrical model and the relative error on the sensitivity along the voltage profile.

\subsection{High Load $Q$}

This scenario involves the power flow variation in a feeder section. In order to monitor a large amount of uncompensated reactive demand $Q$, a test was performed where the feeder extreme (buses 13 to 18 ) had a demand equal to $10 * \mathrm{Q}_{\text {rated }}$. To evaluate the response of the algorithm, an exercise was performed in which we contemplated a capacity of $0.302 \mu \mathrm{F} / \mathrm{km}$ for a hypothetical case where an underground cable is used for the power-in feed.

We obtained the following results for the voltage deviations in consideration of the capacitive susceptance of the feeder within the presented model.

As can be seen in Figure 15, when the shunt capacity was not included in the voltage deviation modelling, the improved accuracy was smaller than in the case of considering it. The voltage profile was fully affected due to the reactive load at the end of the feeder; however, when using the proposed method, the load produced greater sensitivity and the voltage deviation had a better approximation over the specific feeder section, as shown in Figure 16.

In Figure 17 below, a graph with the behaviour of the voltage relative error at the coupling points along the feeder length (in $\mathrm{km}$ ) is illustrated. Two relative error trends are presented. First, in the line modelling, the voltage sensitive effect does not include the parameter $B_{c}$ (shown in orange). In the second trend, the relative errors of the sensitivity analysis on the common coupling points considering $B_{\mathcal{C}}$ (in blue colour) are shown.

The relative error in the extreme zone of the feeder reached error values of $1.63 \%$ for the traditional method and $0.73 \%$ for the method proposed in $\mathrm{PCC}_{18}$.

It was confirmed that the proximity of the reactive load to the coupling points $\mathrm{PCC}_{12}$, $\mathrm{PCC}_{33}, \mathrm{PCC}_{18}$, and power injections at these points at $10 \mathrm{~km}, 14 \mathrm{~km}$, and $24 \mathrm{~km}$ respectively, from the substation, improve the voltage magnitude through the proposed method considering capacitive susceptance in the sensitivity modelling. It is observed that the relative errors decrease from $1.38 \%$ to $0.29 \%$ for $\mathrm{PCC}_{12}$, from $0.81 \%$ to $0.05 \%$ for $\mathrm{PCC}_{33}$, and from $1.63 \%$ to $0.28 \%$ for $\mathrm{PCC}_{18}$. Furthermore, with respect to $\mathrm{PCC}_{6}$, despite not having a high reactive load on nearby buses, it also shows a decrease in its relative error values as a result of the sensitivity analysis. 


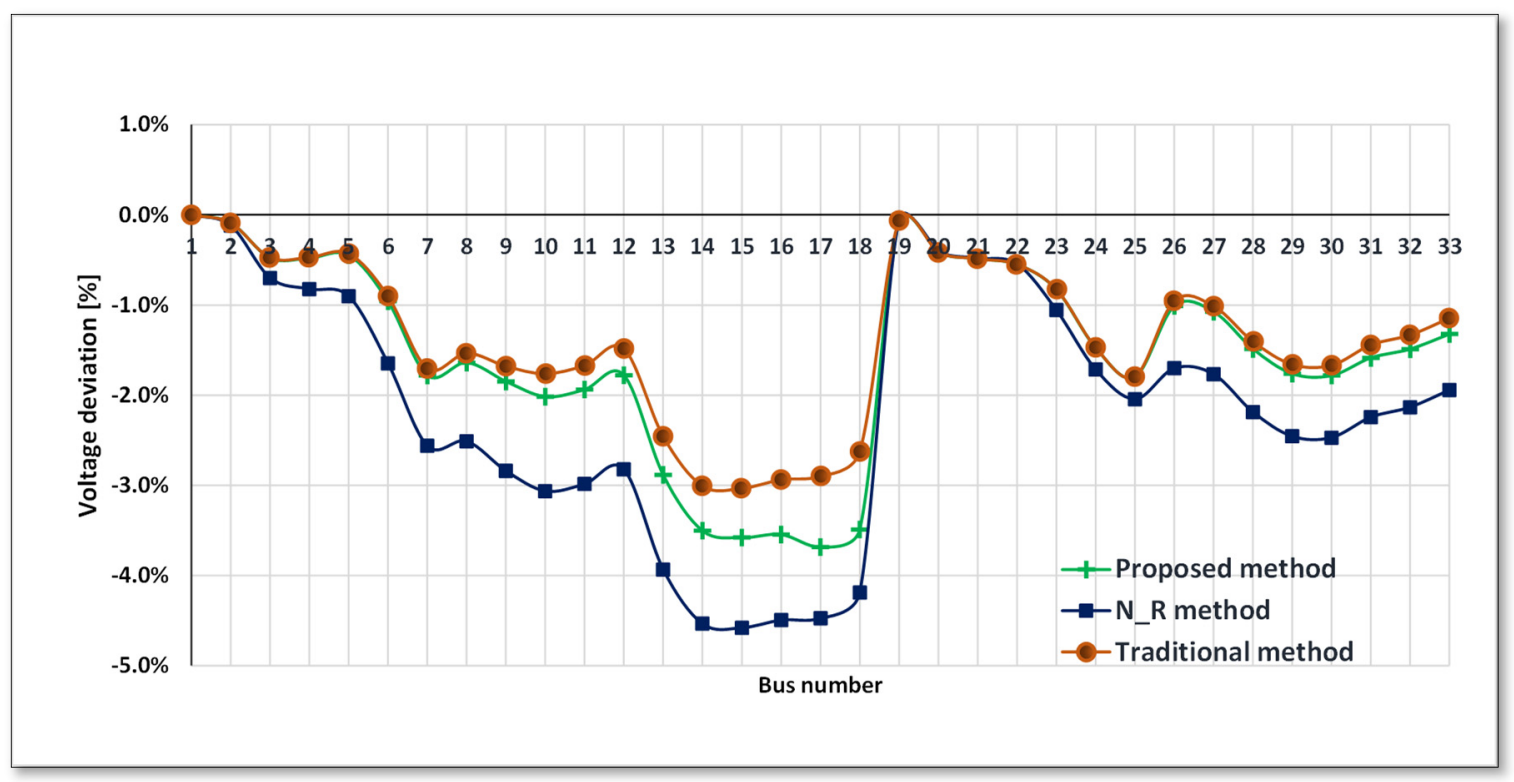

Figure 15. Test high- $Q$ loading in the feeder extreme zone without the line parameter $B c$.

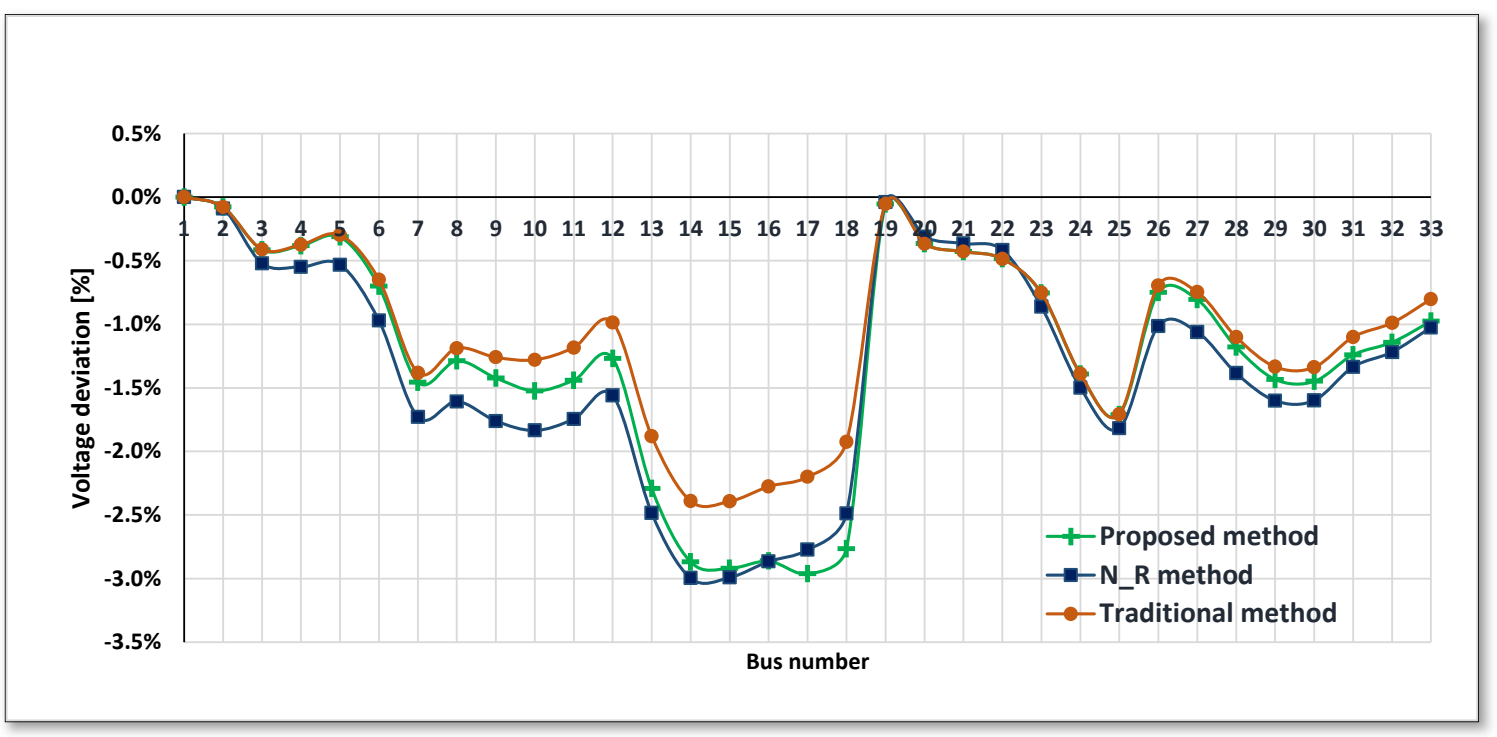

Figure 16. Test high- $Q$ loading in the feeder extreme zone considering the line parameter $B c$.

From the analytical modelling, we found the following: (i) the analytical improvement of the linear sensitivity for voltage control due to power injections from PCCs along the distribution feeders; (ii) linearised complete formulation for the module of voltage deviations between two network buses; iii) non-negligible considerations of the electrical model to operate active distribution networks; iv) a reduction in the computational time and effort because it is a direct technique that improves the approximations to the voltage profile from an existing scenario; and v) a reduction in the relative error with regard to traditional mathematical models for voltage control using the linear sensitivity of the network with DER. 


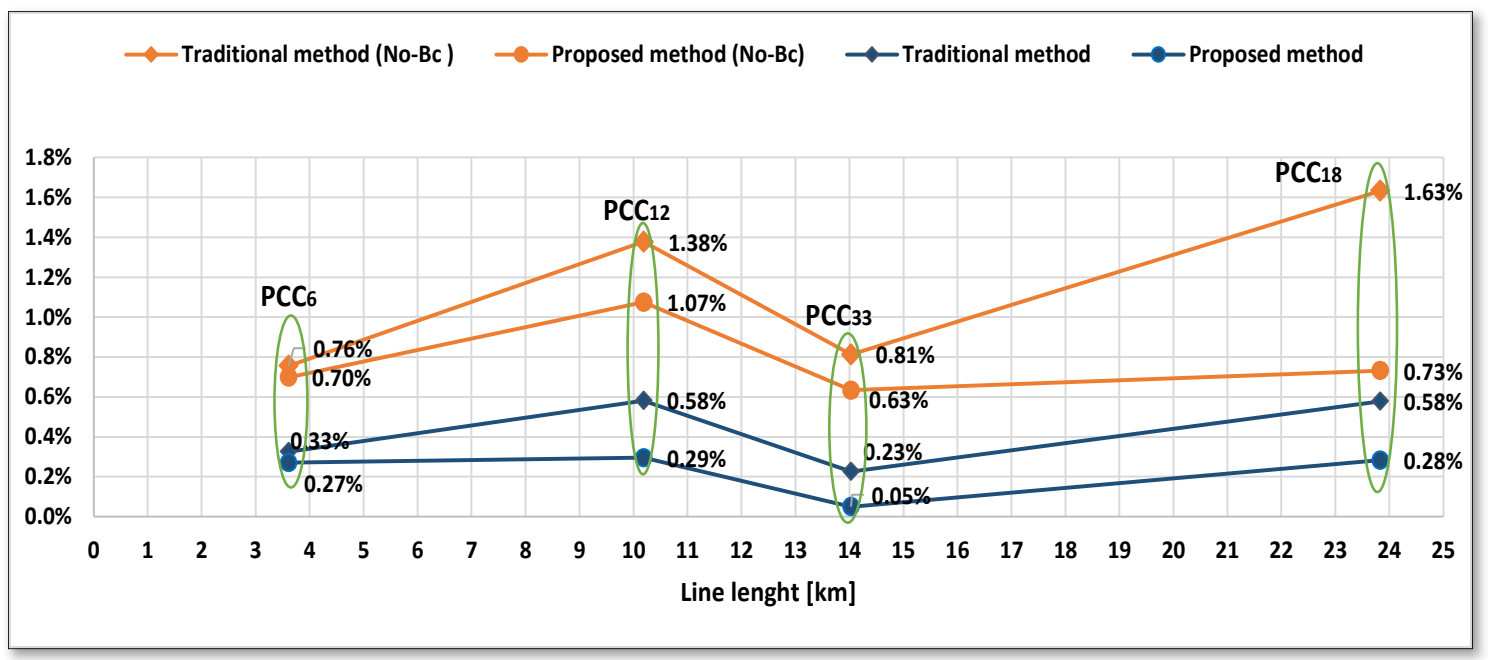

Figure 17. Relative errors at the PCCs.

\section{Conclusions}

In this paper, we have presented a flexible and comprehensive direct methodology for modelling the linear sensitivity of the voltage control in power systems. The proposed methodology is a flexible analytical tool that contemplates the role of a complete electrical model to build a matrix-enhanced voltage profile for system operation. Clearly, the proposed method of linear sensitivity worked best with approximations of the load flow results with power injections along the feeder. The analysis was tested with $Q-V$ compensation in order to control the voltage profile in the new operating scenario with DER.

The application of the method is versatile and direct, and we found that it always improved the voltage profile. Although the analysis was applied to medium-voltage networks, simplified linear models can be extracted from the operating requirements of the analysed system and compensation needs as starting points, while observing the initial voltage conditions of the steady state in buses with self-consumption within a topology close to the voltage control points on the feeder.

As part of future research, it will be possible to use an algorithm for the optimization of the controllable variables inside the objective function, and it will be possible to explore the operational flexibility against the operating constraints for DER, and to investigate the dynamic load modelling and the effects of power injections on other useful ancillary services for the DSO and analytical solutions for the problem distribution system state estimation (DSSE).

Author Contributions: Conceptualization, U.D.L.-M., M.Á.Z. and J.F.M.; formal analysis, U.D.L.-M., M.Á.Z. and J.F.M.; investigation, U.D.L.-M., M.Á.Z. and J.F.M.; methodology, U.D.L.-M., M.Á.Z. and J.F.M. supervision, M.Á.Z. and J.F.M.; writing-review and editing, U.D.L.-M., M.Á.Z. and J.F.M. All authors have read and agreed to the published version of the manuscript.

Funding: This work was partially supported by a scholarship from the High-level Training Program awarded to the first author (Project $N^{\circ}$ PFAN 034-2015) and the authors' own resources.

Acknowledgments: The authors express special thanks to CODENSA ESP by its supporting information to the research project, DIgSILENT GmbH by provide the Thesis Licence PowerFactory.

Conflicts of Interest: The authors declare no conflict of interest.

\section{References}

1. Luhnmann:, T.; Wieben, E.; Treydel, R.; Stadler, M.; Kumm, T. An approach for cost-efficient grid integration of distributed renewable energy sources. Engineering 2015, 1, 447-452. [CrossRef]

2. Olivella-Rosell, P.; Lloret-Gallego, P.; Munné-Collado, Í. Local Flexibility Market Design for Aggregators Providing Multiple Flexibility Services at Distribution Network Level. Energies 2018, 11, 822. [CrossRef] 
3. Mahmud, N.; Zahedi, A. Review of control strategies for voltage regulation of the smart distribution network with high penetration of renewable distributed generation. Renew. Sustain. Energy Rev. 2015, 64, 582-595. [CrossRef]

4. Bollen, M.; Hassan, F. Integration of Distributed Generation in the Power System; Institute of Electrical and Electronics Engineers: Hoboken, NJ, USA, 2011.

5. Ismael, S.M.; Aleem, S.H.A.; Abdelaziz, A.Y.; Zobaa, A.F. State-of-the-art of hosting capacity in modern power systems with distributed generation. Renew. Energy 2019, 130, 1002-1020. [CrossRef]

6. Kacejko, P.; Adamek, S.; Wydra, M. Optimal voltage control in distribution networks with dispersed generation. In Proceedings of the 2010 IEEE PES Innovative Smart Grid Technologies Conference Europe (ISGT Europe), Gothenburg, Sweden, 11-13 October 2010.

7. Mahmud, M.; Hossain, M.; Pota, H.; Nasiruzzaman, B. Voltage Control of Distribution Networks with Distributed Generation using Reactive Power Compesation. In Proceedings of the IECON 2011-37th Annual Conference of the IEEE Industrial Electronics Society, Melbourne, Australia, 7-10 November 2011.

8. Zad, B.B.; Hasanvand, H.; Lobry, J.; Vallée, F. Optimal reactive power control of DGs for voltage regulation of MV distribution systems using sensitivity analysis method and PSO algorithm. Electr. Power Energy Syst. 2015, 68, 52-60.

9. EPRI. Impact Factor, Methods and Considerations for Calculations and Applying Hosting Capacity; EPRI: Palo Alto, CA, USA, 2018; p. 3002011009.

10. Tan, W.-S.; Hassan, M.Y.; Majid, M.S.; Rahman, H.A. Optimal distributed renewable generation planning: A rewiew of diferents approach. Renew. Sustain. Energy Rev. 2013, 18, 626-645. [CrossRef]

11. GE Corporate Research and Development, DG Power Quality. Protection and Reliability Case Studies; NREL: Niskayuna, NY, USA, 2003.

12. Scott, N.; Atkinson, D.J.; Morrel, J. Use of load control to regulate voltage on distribution networks with embedded generation. IEEE Trans. Power Syst. 2002, 17, 510-515. [CrossRef]

13. Kersting, W.H. Distribution System Modeling and Analysi; The Electric Power Engineering Series: Boca Raton, FL, USA, 2002.

14. CIGRE/ JWG C6/B5.25/CIRED. Control and Automation Systems for Electricity Distribution Networks(EDN) of the Future; Electra Cigré; CIGRE: Paris, France, 2017.

15. Abil'Wafa, A. Optimal capacitor allocation in radial distribution systems for loss reduction: A two stage method. Electr. Power Syst. Res. 2012, 95, 168-174. [CrossRef]

16. Rao, R.S.; Narasimham, S.; Ramalingaraju, M. Optimal capacitor placement in a radial distribution system using Plant Growth Simulation Algorithm. Electr. Power Energy Syst. 2011, 33, 1133-1139. [CrossRef]

17. Putra, J.T.; Sarjiya. Reactive Power Optimization of distributed generation for voltage regulation of distribution systems. In Proceedings of the 2016 8th International Conference on Information Technology and Electrical Engineering (ICITEE), Yogyakarta, Indonesia, 5-6 October 2016.

18. Zeng, X.-J.; Tao, J.; Zhang, P.; Pan, H.W.Y. Reactive Power Optimization of Wind Farm based on Improved Genetic Algoritm. Energy Procedia 2012, 14, 1362-1367. [CrossRef]

19. Engelhardt, S.; Erlich, I.; Feltes, C.; Kretschmann, J.; Shewarega, F. Reactive power capability of wind turbines based on doubly fed induction generators. IEEE Trans. Energy Convers. 2011, 26, 364-372. [CrossRef]

20. Pollock, J.; Hill, D. Application of Distributed Generation Reactive Power Control Modes to Increase System Stability. In Proceedings of the CIRED, Helsinki, Finland, 14-15 June 2016.

21. CIGRE. 586. WG C6.24. Capacity of Distribution Feeders for Hosting DER; Electra Cigré; CIGRE: Paris, France, 2014.

22. Khatod, D.K.; Pant, V.; Sharma, J. A Novel Approach for Sensitivity Calculations in the Radial Distribution System. IEEE Trans. Power Deliv. 2006, 21, 2048-2057. [CrossRef]

23. Wei, W.; Xu, L.; Shidan, W. Research on the reactive power optimization of distribution network including DG. In Proceedings of the 3rd IEEE International Symposium on Power Electronics for Distributed Generation Systems (PEDG), Aalborg, Denmark, 28 June-1 July 2012.

24. Ahmadi, H.; Martí, J.R.; Dommel, H.W. A framework for Volt-VAR optimization in Distribution Systems. Trans. Smart Grids 2015, 6, 1473-1783. [CrossRef]

25. Griet, D.; Shariatzadeh, F.; Srivastava, A. Volt/VAr Optimization with energy savings for distribution system using intelligent control. In Proceedings of the North. American Power Symposium (NAPS), Manhattan, KS, USA, 22 September 2013.

26. Teng, J.-H. A direct approach for distribution system load flow solutions. IEEE Trans. Power Deliv. 2003, 18, 882-887. [CrossRef]

27. Lynn, P. Power System Load Flow Analysis; Mc Graw-Hill: New York, NY, USA, 2005.

28. Brahmendra, G.V.; Palanisamy, K. A review of energy storage participation for ancillary services in a microgrid environment. Inventions 2020, 5, 63 .

29. Iorgulescu, M.; Ursu, D. Reactive Power Control and Voltage Stability in Power Systems. In Reactive Power in AC Power Systems; Springer: Cham, Switzerland, 2017; pp. 227-248.

30. Bakhshideh, B.; Lobry, Z.J.; Vallée, F. A centralized approach for voltage control of MV distribution systems using DGs power control and a direct sensitivity analysis method. In Proceedings of the IEEE International Energy Conference (ENERGYCON), Leuven, Belgium, 8 April 2016.

31. Conti, S. Voltage Sensitivity Analysis in MV Distribution Networks. In Proceedings of the International Conference on Electric Power Systems, High Voltages, Electric Machines, Tenerife, Spain, 16-18 December 2006. 
32. Kumar injeti, S.; Kumar, N.P. A novel approach to identify optimal access point and capacity of multiple DGs in a small, medium and large scale radial distribution systems. Electr. Power Energy Syst. 2013, 45, 142-151. [CrossRef]

33. Lakervi, E.; Holmes, E.J. Electricity Distribution Network Design, 2nd ed.; IEE Power Series 21: London, UK, 1998.

34. Carvalho, P.M. Distributed Reactive Power Generation Control for Voltage Rise Mitigation in Distribution Networks. IEEE Trans. Power Syst. 2008, 23, 766-772. [CrossRef]

35. Wang, M.; Yi, H.; Yang, Z.; Tao, R.; Liu, X.; Zhuo, F.; Hu, X. Comprehensive Control of Voltage Quality in Distribution Network based on Reactive Power Optimization. In Proceedings of the 2020 IEEE 9th International Power Electronics and Motion Control. Conference (IPEMC2020-ECCE Asia), Nanjing, China, 32 May-3 June 2020.

36. Patari, N.; Srivastava, A.K.; Qu, G.; Li, N. Distributed Optimal Voltage Control for Three Phase Unbalanced Distribution Systems with DERs. In Proceedings of the 2020 IEEE Industry Applications Society Annual Meeting, Detroit, MI, USA, 3-4 March 2020.

37. Matei, G.G.; Neagu, B.C.; Gavrilaş, M. Optimal Voltage Control Based on a Modified Line Drop Compensation Method in Distribution Systems. In Proceedings of the 2018 IEEE International Conference on Environment and Electrical Engineering and 2018 IEEE Industrial and Commercial Power Systems Europe (EEEIC/I\&CPS Europe), Palermo, Italy, 6 June 2018.

38. Xu, H.; Dominguez-García, A.; Veeravali, V.; Sauer, P. Data-driven Voltage Regulation in Radial Power Systems. Trans. Power Syst. 2020, 35, 2133-2143. [CrossRef]

39. Peschon, J.; Piercy, D.S.; Tinney, W.F.; Tveit, O.J. Sensitivity in Power Systems. IEEE Trans. Power Appar. Syst. 1968, 87, 1687-1696. [CrossRef]

40. Baran, M.E.; Wu, F.F. Network reconfiguration in distribution systems for loss reduction and load balancing. IEEE Trans. Power Deliv. 1989, 4, 1401-1407. [CrossRef] 